\title{
Towards online triggering for the radio detection of air showers using deep neural networks
}

\author{
Florian Führer ${ }^{1,2, \star}$, Tom Charnock ${ }^{1}$, Anne Zilles ${ }^{1}$, and Matias Tueros ${ }^{3}$ \\ ${ }^{1}$ Sorbonne Université, UPMC Univ. Paris 6 et CNRS, UMR 7095, Institut d'Astrophysique de Paris, 98 bis \\ bd Arago, 75014 Paris, France \\ ${ }^{2}$ Sorbonne Université, Institut Lagrange de Paris, 98bis boulevard Arago, 75014 Paris, France \\ ${ }^{3}$ Instituto de Física La Plata - CONICET/CCT- La Plata. Calle 49 esq 115. La Plata, Buenos Aires, Argentina
}

\begin{abstract}
The detection of air-shower events via radio signals requires the development of a trigger algorithm for clean discrimination between signal and background events in order to reduce the data stream coming from false triggers. In this contribution we will describe an approach to trigger air-shower events on a single-antenna level as well as performing an online reconstruction of the shower parameters using neural networks.
\end{abstract}

\section{Introduction}

Machine learning and, in particular, deep learning using neural networks (NN) is a field which is currently beginning to thrive in astronomy. This means we can make use of well-established techniques to tackle the challenge of both self-triggering on radio signals, preferably on the single antenna level, and performing online parameter-reconstruction of neutrino and ultra high energy cosmic ray (UHECR) induced air-shower events based on detected signals in an array of antennas. There is expected to be a large amount of human-made background noise (mostly transient noise) in the radio-data which needs to be dismissed efficiently to distinguish between signals from air showers induced by cosmic primaries and neutrino events. As an example of the scale of the problem, we expect $\sim 1$ neutrino event-per-year for one of the proposed 10000 antennas sub-arrays of the GRAND experiment [1], while the trigger rate due to background will be $\sim \mathrm{kHz}$.

Through the use of machine learning we propose that the false event detection rate can be reduced significantly, which reduces the data-stream rate and effectively lowers the triggering threshold. Lowering the triggering threshold would allow less-energetic air showers to be detected which would lead to an important gain in statistics. The feasibility of self-triggering on air-shower events via the measurement of the radio signals is reinforced by the reports of $[2,3]$. The purpose of this proceeding is to demonstrate the feasibility of $\mathrm{NN}$ based triggering and to motivate further research in this direction.

\section{Neural networks as triggers}

We assume the simplified two step data taking process of a radio array depicted in Fig. (1). The process starts with a trigger on a single antenna level, and ends by the reconstruction of the shower parameters. In this proceeding we will describe a NN trigger which searches the data recorded from the antenna in a given time interval for a signal. In a future publication we will describe the reconstruction network which takes the collected time traces containing signals from all antennas as an input and reconstructs the primary particle's type and parameters.

\subsection{Deep learning}

In machine learning, "deep learning" allows for abstraction between the output and the provided input data where the non-trivial function mapping the input to the output is fitted [4]. Since the neural

^e-mail: fuhrer@iap.fr

(C) The Authors, published by EDP Sciences. This is an open access article distributed under the terms of the Creative Commons Attribution License 4.0 (http://creativecommons.org/licenses/by/4.0/). 


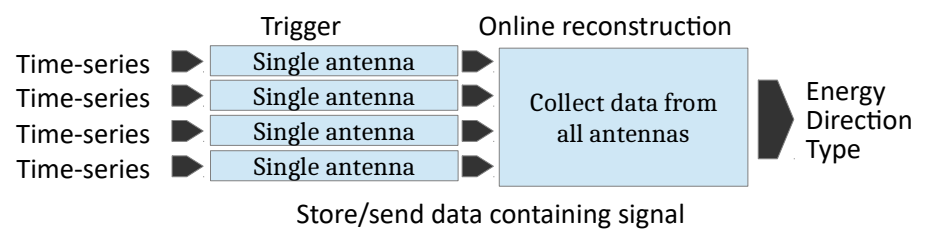

Figure 1. A sketch of the considered pipeline of online triggering and reconstruction. At each antenna a NN searches the incoming data stream for a radio signal. The discovered signals are passed together with a time stamp to a second $\mathrm{NN}$, which can then perform the online reconstruction of the shower parameters.

network learns directly from the data that is provided, the only necessary products are simulations of the real data. Provided with such simulations we expect a NN based trigger to better generalise than other methods, such as matched filters that necessitate templates for each event type. As an additional advantage, a NN based trigger will have a lower computational cost than using matched filters [5].

Training data: To demonstrate the feasibility of online triggering using NN, we consider a GRANDlike toy array consisting of $35 \times 35$ antennas on slope of $5^{\circ}$. Our simulated signals consist of three independent voltage trace components which are folded with the antenna response and Gaussian white noise with a standard deviation of $15 \mu \mathrm{V}$ and then filtered to $50-200 \mathrm{MHz}$. For the UHECR we use protons and iron-nuclei with an energy of $0.1-100 \mathrm{EeV}$ and a zenith angle of $65-85^{\circ}$. The neutrino distribution in direction and energy follow the expected distribution for the GRAND array.

Some notes on the generalisation to real data: A NN trained on white noise is likely to have a similar performance on realistic noise if the network can adapt to the realistic noise. Thanks to transfer learning, where a network fully trained on simulations is essentially tuned by training with a small amount of real data, the network adaptation is possible without retraining from scratch [6]. Given that the network can learn about realistic noise, there is still the obstacle of transient noise closely mimicking real signals. This does not pose a problem as long as the transient noise can be measured and hence learned, allowing the network to distinguish between the minute differences between different samples of data. Given that we do not expect most transient signals to exactly mimic true signals we are confident that the network will be able to filter most transient noise ${ }^{1}$.

\section{Results}

For the trigger, we trained a convolutional $\mathrm{NN}$ with three convolutional layers and one fully connected hidden layer with 64 units. The convolutional layers have consecutive kernels sizes of 16, 8 and 8 and 16, 32 and 64 filters and we perform a max-pooling over consecutive time elements. We train the network by minimising the categorical cross entropy. The neural network is written using TensorFlow for its inter-platform and co-GPU and -CPU portability.

The NN is trained using 150000 time traces half containing a signal and half containing only noise. We found that the number of correctly classified traces using the $\mathrm{NN}$ is $72 \%$, which is higher than when using a threshold trigger at $60 \mu \mathrm{V}$ applied to each of the three voltages traces independently, where the accuracy is $69 \%$. While the true positive classification is only slightly improved by the NN, $43 \%$ compared to $42 \%$, the number of false triggers are reduced by more than one order of magnitude, from $4 \%$ to less than $0.2 \%$. Since we wish to reduce the data-stream efficiently it is exactly the rate

\footnotetext{
${ }^{1}$ We expect to profit from the fact that the use of polarization information is already built into the NN architecture.
} 
of false events that we want to reduce. A detailed comparison of the two trigger algorithms is beyond the scope of this proceeding, we postpone this together with an accurate calculation of the expected data stream to a future publication.

\section{Conclusion and outlook}

In this proceeding we have described the first steps towards self-trigger on radio signals using a NN. This NN-based trigger performs better than a conventional threshold trigger, in particular reducing the number of false alarms drastically and therefore potentially making the data-stream of signal detections more pure. Our promising results motivate further exploration of the existing potential to improve the NN trigger. Of course the $\mathrm{NN}$ can be taught to better generalise through the gathering of more data or perform better via hyperparameter tuning, but another possibility is to split the trigger into two levels. The first level of a two level trigger algorithm would be the $\mathrm{NN}$ described in this proceeding and the second level would use the coincidences in the arrival time among nearby antennas to further reduce the false event rate. However, if this second level trigger is built into the online reconstruction of the particle shower using the an entire GRAND sub-array, the accidental over-triggering due to noise can essentially be absorbed into the reconstruction network (mentioned earlier) which is inherently more resilient to artefacts due to the way it correlates all antennas. A complementary, and perhaps more simple, modification to the level-one trigger could be to train the NN specifically to do online denoising of voltage traces directly from the observed traces.

Even though the proposed network seems much more computationally heavy than a simple threshold trigger, advancements in low power, highly optimised machine learning chipsets actually suggests that there will be little to no difference in computational complexity hence making the process of NN-based triggering scalable.

\section{Acknowledgement}

This work has received support from the French Agence Nationale de la Recherche under the references ANR-10-LABX-63, ANR-11-IDEX-0004-02 and ANR-16-CE31-0001. This work has made use of the Horizon Cluster hosted by the Institut d'Astrophysique de Paris. The Quadro P6000 used in this study was donated by the NVIDIA Corporation.

\section{References}

[1] K. Fang et al., PoS ICRC2017, 996 (2017)

[2] Le Coz, A. et al., PoS ICRC2017 406 (2017)

[3] The Pierre Auger Collaboration, JINST 711023 (2012)

[4] Ian Goodfellow and Yoshua Bengio and Aaron Courville, Deep Learning (MIT Press, 2016)

[5] Daniel George and E. A. Huerta, Phys. Rev. D 97, 044039 (2018)

[6] Sinno Jialin Pan and Qiang Yang, IEEE Transactions on Knowledge and Data Engineering vol. 22, no. 10, 1345 (2010) 